\title{
Optical observations of GRB 060218/SN 2006aj and its host galaxy
}

\author{
Patrizia Ferrero*, Eliana Palazzi ${ }^{\dagger}$, Elena Pian** and Sandra Savaglio ${ }^{*}$ \\ ${ }^{*}$ Thüringer Landessternwarte Tautenburg, D-07778 Tautenburg, Germany \\ ${ }^{\dagger}$ INAF - Istituto di Astrofisica Spaziale e Fisica Cosmica di Bologna, I-40129 Bologna, Italy \\ **INAF - Osservatorio Astronomico di Trieste, I-34131 Trieste, Italy \\ ${ }^{\ddagger}$ Max-Planck-Institut für Extraterrestrische Physik, D-85748 Garching, Germany \\ (on behalf of a larger collaboration)
}

\begin{abstract}
The supernova SN 2006aj associated with GRB 060218 is the second-closest GRB-SN observed to date $(z=0.033)$ and is the clearest example of a SN associated with a Swift GRB with the earliest optical spectroscopy. Its optical data showed that this is the fastest evolving and among the least luminous GRB-SNe (70\% as luminous as SN1998bw). However, its expansion velocity and a comparison with other stripped-envelope SNe suggest that SN2006aj is an intermediate object between Type Ic GRB-SNe and those not accompained by a GRB. High-resolution optical spectroscopy together with SDSS pre-burst observations revealed that the host galaxy of SN2006aj is a low-luminosity, metal-poor star-forming dwarf galaxy.
\end{abstract}

Keywords: Gamma-ray Bursts, Supernovae, GRB 060218, SN 2006aj

PACS: $98.70 . \mathrm{Rz}, 97.60 . \mathrm{Bw}$

\section{INTRODUCTION}

The first hint for an association of long-duration GRBs with core-collapse supernovae of Type Ic [1] came with the contemporaneous discovery of GRB 980425 and a local SN (1998bw) in its error circle [2]. Subsequently, the first spectroscopic identification of a SN Ic superposed on a GRB afterglow (GRB 030329/SN 2003dh; e.g. [3]) revealed the unambiguous signatures of this connection. This event was followed by GRB 031203/SN 2003lw (e.g. [4]). Almost three years later a new spectacular case of a nearby GRB-SN association was found: GRB 060218/SN 2006aj.

On 2006 February 18, at 03:34:30 UT, the Burst Alert Telescope (BAT) on board the Swift satellite detected the bright GRB 060218 [5]. The Swift X-ray Telescope (XRT) and the UV/Optical Telescope (UVOT) also detected its afterglow in the X-ray and optical bands [5, 6], respectively, leading to a precise localization of the optical counterpart [7]. Due to its unusual properties in the gamma-ray band [8] and its odd behavior [9], its nature was not clear till the determination of its redshift $(z=0.033$ [10] $)$ and the discovery of an association with a supernova [11]. GRB 060218 is classified as an X-ray Flash [8], with $E_{\text {iso }}$ comparable with other GRB-SNe and consistent with the Amati relation [12]. It is probably not an off-axis event [13].

The low redshift and the brightness of the object allowed extensive follow-up observations with ground-based facilities [14, 15, 16, 17, 18, 19].

Here, we summarize results from a photometric and spectroscopic ESO Very Large Telescope (VLT) campaign on GRB 060218/SN 2006aj, with additional data gathered 
with the robotic Liverpool Telescope (LT), the Lick Observatory Shane 3m Telescope and the robotic Katzman Automatic Imaging Telescope (KAIT; [20, 21]). (For a more detailed description of the observations and of the data reduction see [17, 18], and references therein.) In addition we discuss the properties of the host galaxy.

\section{OBSERVATIONS}

The data set includes observations performed over a period of almost 4 weeks by means of different telescopes [17, 18]:

- VLT- We observed GRB 060218/SN 2006aj both spectroscopically [17] and photometrically [18] with the ESO $8.2 \mathrm{~m}$ VLT equipped with the FORS1 and the FORS2 instruments nearly daily from February 21 until 26 days after the burst. Near the epoch of the supernova maximum, we obtained a high-dispersion spectrum with VLT/UVES.

- Liverpool Telescope- Several data were acquired over a period of about 2.5 weeks post-burst by the $2 \mathrm{~m}$ robotic Liverpool Telescope on La Palma.

- Shane and KAIT Telescopes at Lick Observatory- An additional spectrum was obtained with the Shane $3 \mathrm{~m}$ telescope 4 days after the burst and other photometric observations were coordinated at the $0.8 \mathrm{~m}$ robotic KAIT telescope during four consecutive nights between 3 to 6 days after the burst.

As the host galaxy and SN 2006aj were not separated at the angular resolution of the images, the host magnitudes (for details see [18] and references therein) have been subtracted from all data. For the extinction correction, we used the values derived by [22] for our Galaxy and the host galaxy, $A_{V}=0.39 \mathrm{mag}$ and $A_{V}=0.13 \mathrm{mag}$, respectively.

\section{RESULTS AND DISCUSSION}

\section{The spectra}

The spectra of SN 2006aj [17, 23], with their broad absorption lines and their lack of hydrogen and helium features (Fig. 1), clearly resemble those of broad-lined Type Ic supernovae. In particular, the deduced properties of SN 2006aj are similar to those of the non-GRB supernova SN 2002ap [24]: small released energy and small ejected mass [23]. These characteristics, along with the softness and the extremely long duration $(2000 \mathrm{~s}$, [25]) of GRB 060218, suggest that this event is different from other GRB-SNe known so far. One critical parameter (see [23] for a detailed modelling) could be the initial mass of the progenitor star: it may have been significantly smaller for SN 2006aj $\left(\sim 20 M_{\odot}\right)$ than for other GRB-SNe $\left(\sim 40 M_{\odot}\right)$. Moreover, the collapse/explosion released less energy. A star of mass $\sim 20 M_{\odot}$ is expected to form a neutron star rather than a black hole when its core collapses, possibly resulting in a highly magnetized neutron star (a magnetar) [23]. 


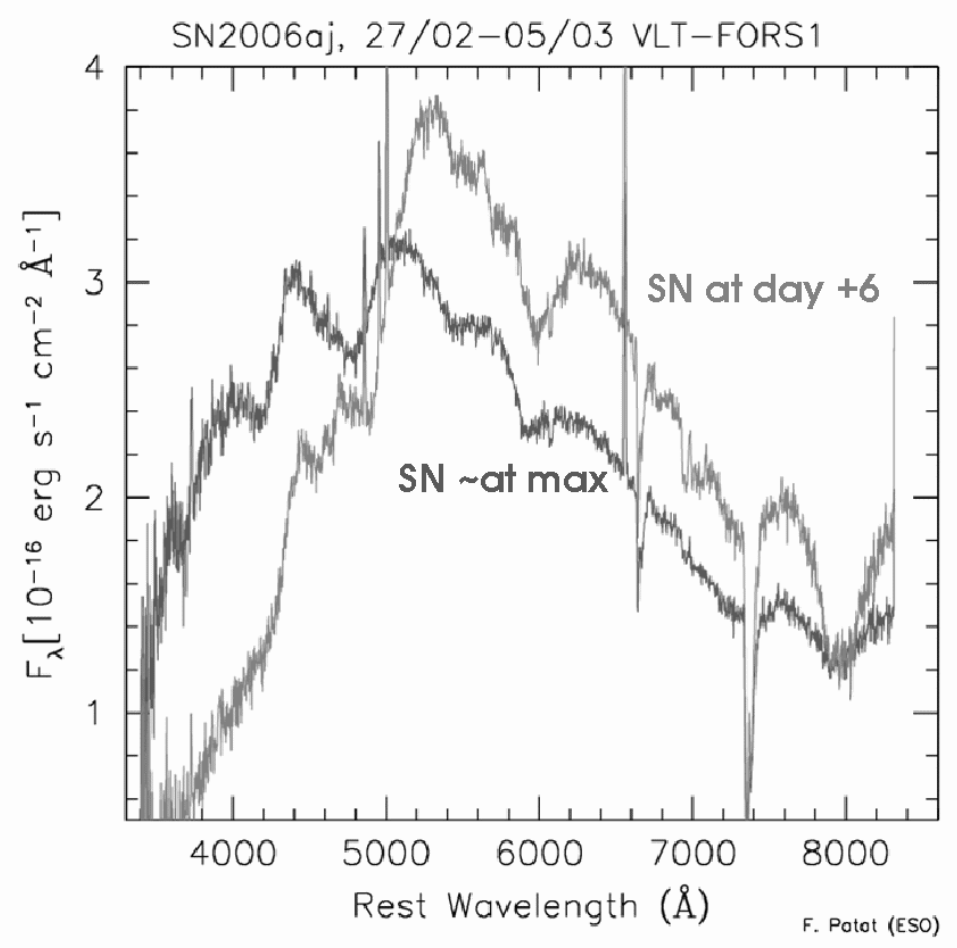

FIGURE 1. VLT low-resolution spectra of SN 2006aj acquired at its maximum (February 27) and six days later (March 5). The strong evolution toward the red is common of Type Ic broad-lined SNe and suggests a strong expansion velocity.

\section{The light curves}

Following [26, 27], we modeled the light curves of SN 2006aj using SN 1998bw as a template that was shifted to the corresponding redshift and scaled in luminosity (in the SN rest frame) by a factor $k$ and in time evolution by a factor $s$, while zero host extinction was assumed for SN 1998bw [28]. In doing so, we find an additional component visible in the early data that makes the light curve systematically brighter than that of SN 1998bw (Fig. 2]). This component has also been noted by [15] and explained as the light due to a shock break-out through a dense progenitor wind [8].

It is interesting to compare the properties of SN 2006aj with those of other Type Ic supernovae. Fig. 3 shows that the three well-observed, low-redshift GRB-SNe (SN 1998bw, SN 2003dh and SN 2003lw) are very similar with respect to their light curves. On the other hand, SN 2006aj at maximum light is fainter by about a factor of two, but it is still a factor of 2-3 more luminous than the other broad-lined Type Ic supernovae not related to GRBs (see also Fig. 6 in [18]).

Normal Type Ic supernovae rise to a peak in approximately 10-12 days. Previously known GRB-SNe showed a longer rise time (14-15 days), but SN 2006aj rose as fast as normal Type Ic SNe and also declined fast.

SN 2006aj appears to be an intermediate object between GRB-SNe and other Type Ic SNe without a detected GRB. 


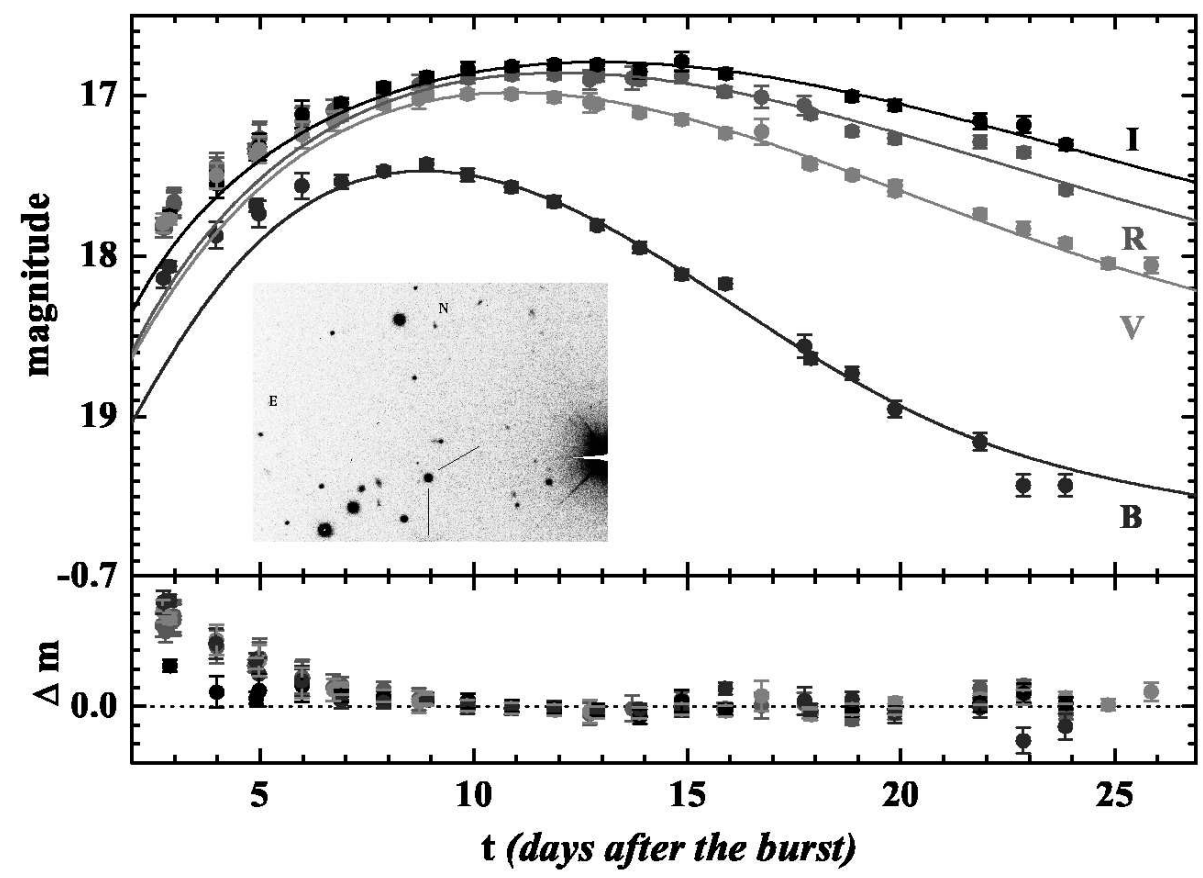

FIGURE 2. The BVRI light curves of SN 2006aj. The data have been corrected for extinction and host flux contribution. The fit has been done using the light curves of SN 1998bw as a template and not considering the data that were taken before 8.8 days post burst. The residuals $\Delta m$ are the difference between the observed values and the fit. The inset shows a VLT/FORS2 R-band image of SN 2006 aj. Revised from [18].

\section{The host galaxy}

The host galaxy of GRB 060218/SN 2006aj was imaged pre-burst by the Sloan Digital Sky Survey [29]. These images were used to obtain its magnitudes [18], as the SN brightness was hiding the host component, and to measure its diameter $(\sim 2 \mathrm{kpc}$, for $z=0.033$ ).

The VLT/UVES spectrum allowed a measurement of the total extinction toward SN 2006aj and it turned out that the main contribution is from our Galaxy $(E(B-V)=$ $0.127 \pm 0.005)$, while $E(B-V)_{\text {host }}$ is only $0.044 \pm 0.001$ [22]. The latter is supported by the SED of the galaxy (see Fig. 4).

In addition, spectroscopic studies led to an estimate of the $S F R \sim 0.07 M_{\odot} /$ year and of the metallicity $Z \sim 0.07 Z_{\odot}[30]$. Taking into account slit losses, the $S F R$ might be understimated by a factor 3. This would imply that the SN 2006aj host has a $S F R$ comparable to that of the Milky Way, while its stellar mass is about 1000 times smaller $\left(M_{\text {host }}=10^{7.2 \pm 0.3} M_{\odot}\right)[31]$.

\section{ACKNOWLEDGMENTS}

F.P. thanks Sylvio Klose for useful advice and comments and acknowledges support by DFG grant Kl 766/13-2 and by the German Academic Exchange Service (DAAD) under 


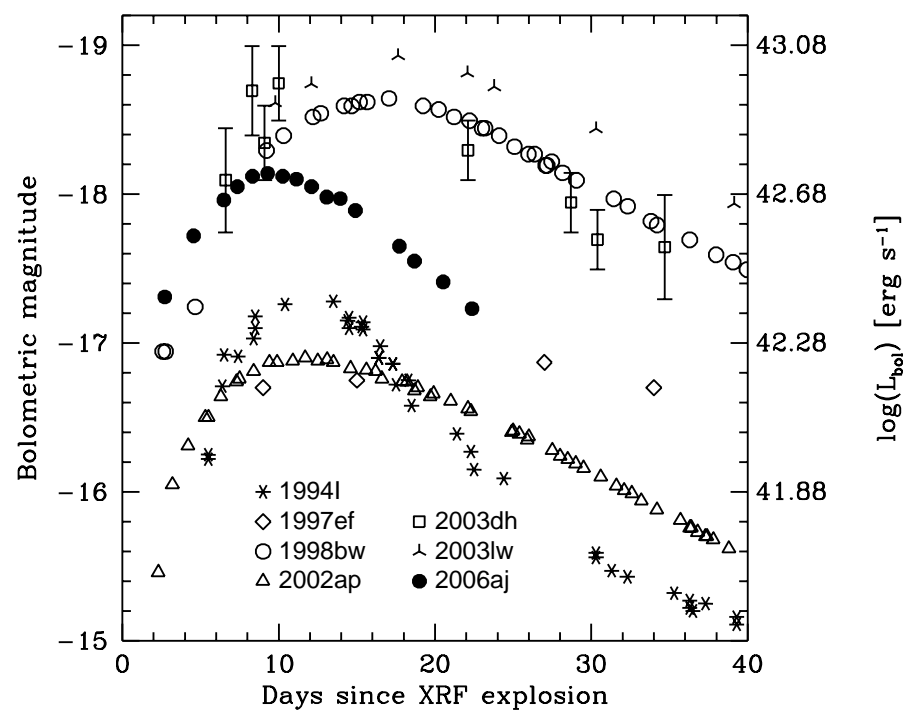

FIGURE 3. A comparison between the bolometric light curves of normal Type Ic SNe and GRB-SNe. (For details see also [17].)

grant No. D/05/54048.

\section{REFERENCES}

1. A. V. Filippenko 1997, ARA\&A, 35, 309-355

2. T. J. Galama, P. M. Vreeswijk, J. van Paradijs, et al. 1998, Nature, 395, 670-672

3. K. Z. Stanek, T. Matheson, P. M. Garnavich, et al. 2003, ApJ, 591, L17-L20

4. D. Malesani, G. Tagliaferri, G. Chincarini et al. 2004, ApJ, 609, L5-L8

5. G. Cusumano, S. Barthelmy, N. Gehrels, et al. 2006, GCN, 4775

6. G. Kennea, D. N. Burrows, G. Cusumano, \& G. Tagliaferri 2006, GCN, 4776

7. G. Marshall, S. Immler, \& G. Cusumano 2006, GCN, 4779

8. S. Campana, V. Mangano, A. J. Blustin, et al. 2006, Nature, 442, 1008-1010

9. N. Gehrels 2006, GCN, 4787

10. N. Mirabal, \& J. P. Halpern 2006, GCN, 4792

11. N. Masetti, E. Palazzi, E. Pian, \& F. Patat 2006, GCN, 4803

12. L. Amati, F. Frontera, C. Guidorzi \& E. Montanari 2006, GCN, 4846

13. J. Nousek, G. Cusumano, A. Moretti, et al. 2006, GCN, $\mathbf{4 8 0 5}$

14. M. Modjaz, K. Z. Stanek, P. M. Garnavich, et al. 2006, ApJ, 645, L21-L24

15. N. Mirabal, J. P. Halpern, D. An, J. R. Thorstensen, \& D. M. Terndrup 2006, ApJ, 643, L99-L102

16. J. Sollerman, A. O. Jaunsen, J. P. U. Fynbo et al. 2006, A\&A, 454, 503-509

17. E. Pian, P. A. Mazzali, N. Masetti, et al. 2006, Nature, 442, 1011-1013 

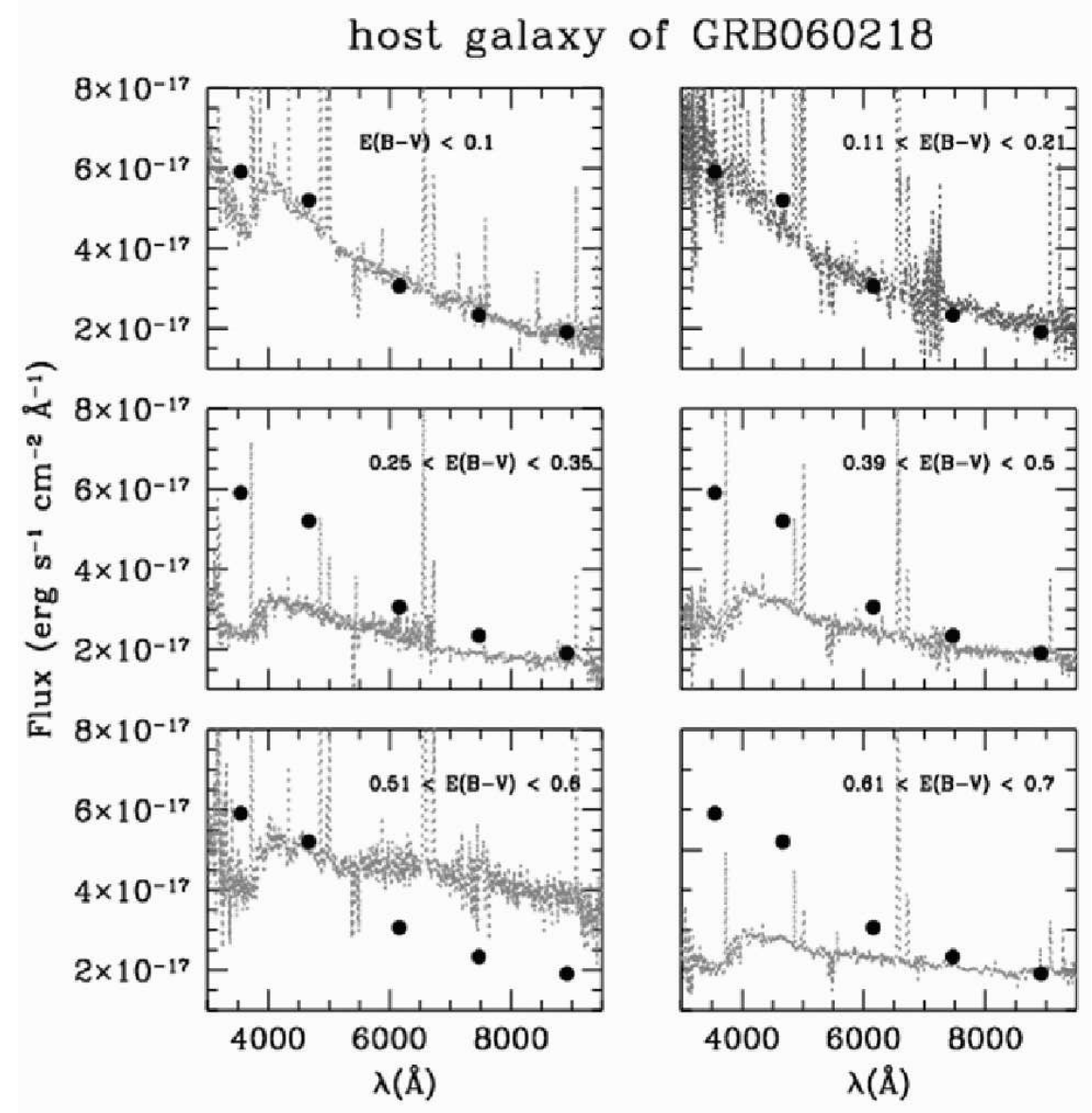

FIGURE 4. The broad-band SED of the host galaxy of SN 2006aj (thick points) is compatible with a moderately absorbed galaxy (starburst templates from [32]). The data have been corrected for galactic extinction.

18. P. Ferrero, D. A. Kann, A. Zeh, et al. 2006, $A \& A, 457,857-864$

19. A. M. Soderberg, S. R. Kulkarni, E. Nakar, et al. 2006, Nature, 442, 1014-1017

20. A. V. Filippenko, W. D. Li, R. R. Treffers, \& M. Modjaz 2001, in Small Telescope Astronomy on Global Scales, edited by W. P. Chen, C. Lemme, \& B. Paczyński, (San Francisco: ASP), 121-130

21. W. Li, A. V. Filippenko, R. Chornock, \& S. Jha 2003, PASP, 115, 844-853

22. E. W. Guenther, S. Klose, P. M. Vreeswijk, E. Pian, \& J. Greiner, GCN, 4863

23. P. A. Mazzali, J. Deng, K. Nomoto, et al. 2006, Nature, 442, 1018-1020

24. R. J. Foley, M. S. Papenkova, B. J. Swift, et al. 2003, PASP, 115, 1220-1235

25. S. Barthelmy, J. Cummings, T. Sakamoto, et al. 2006, GCN, 4806

26. A. Zeh, S. Klose, \& D. H. Hartmann 2004, ApJ, 609, 952-961

27. A. Zeh, S. Klose, \& D. H. Hartmann 2005, in Proc. 22nd Texas Symp. on Relativistic Astrophysics., edited by P. Chen et al. (Stanford:SLAC) (astro-ph/0503311)

28. F. Patat, E. Cappellaro, J. Danziger, et al. 2001, ApJ, 555, 900-917

29. R. J. Cool, D. G. Eisenstein, D. W. Hogg, et al. 2006, GCN, 4777

30. K. Wiersema, et al. 2007, $A \& A$, accepted

31. S. Savaglio, K. Glazebrook, \& D. Le Brogne, in preparation

32. A. L. Kinney, D. Calzetti, R. C. Bohlin, K. McQuade, T. Storchi-Bergmann, \& H. R. Schmitt 1996, ApJ, 467, 38-60 